\section{Bypassing the iPS logjam}

\section{By Kai-Jye Lou, Staff Writer}

Researchers at the Stanford University School of Medicine have found a way to efficiently convert mouse fibroblasts into functional neurons without first reverting the skin cells to a pluripotent state. ${ }^{1}$ The protocol could make it possible to use host-derived fibroblasts to create neurons for disease modeling, drug screening and transplantation studies.

The conventional approach to turning fibroblasts into a different cell type involves reprogramming the skin cells into induced pluripotent stem (iPS) cells using a quartet of transcription factors (Oct4, Sox2, Klf4 and c-Myc). ${ }^{2,3}$ The iPS cells are then isolated, expanded and differentiated into a new cell type. However, the efficiency of the process is low and the procedure can take months.

The Stanford protocol skips the pluripotent step altogether and in doing so gains efficiency and speed (see Figure 1, "Generating neurons from fibroblasts").

In mouse fibroblasts, forced expression of three transcription factors-achaete-scute complex homolog 1 (Ascl1), POU class 3 homeobox 2 (Pou3f2; Brn2) and myelin transcription factor 1-like (Myt1l) - converted the cells into induced neuronal cells without first reverting them to a pluripotent state. The converted cells expressed neuron-specific proteins, generated action potentials and formed functional synapses in vitro.

Most of these neurons were excitatory and expressed markers of cortical identity. However, the researchers also identified a small subpopulation of cells that expressed markers of $\gamma$-aminobutyric acid (GABA)ergic neurons, which are usually inhibitory.

"The surprising findings from our study were that not only was it possible to convert skin cells directly into neurons, but also the process itself was also fast and efficient," said Marius Wernig, an assistant professor of pathology in the Institute for Stem Cell Biology and Regenerative Medicine at Stanford. "It turns out that the expression of three transcription factor genes is sufficient to turn up to $20 \%$ of the starting population of skin cells into neurons in about a week."

Results were published in Nature.

"If you want to go the iPS cell route, it usually takes four to six weeks to first reprogram fibroblasts into pluripotent stem cells," said Wernig, who was corresponding author on the paper. "You then need to characterize the reprogrammed cells to make sure that they are indeed iPS cells. And then you need to differentiate the pluripotent cells into neurons, which is usually another four- to six-week procedure."

"This work provides an alternative approach that also turns out to be remarkably efficient," said Philip Gregory, CSO and VP of research at Sangamo BioSciences Inc. "With iPS cell approaches, $0.1 \%$ efficiency is generally considered successful, though some groups have recently been able to get efficiency into the double digits. If the efficiency of the system is portable to human cells, it could make iPS cell technologies redundant where neurons are the desired cell type."

Although previous studies have shown that it is possible to skip the pluripotent step when directly converting between cells from related lineages, ${ }^{4,5}$ the Stanford team is the first to show direct conversion between unrelated cell lineages.

"Previous studies in muscle cells and pancreatic cells have shown that you could directly convert cells from one type into another, but those studies converted between cells that are derived from the same germ layer," said Sebastian Jessberger, an assistant professor in the Department of Biology at The Swiss Federal Institute of Technology Zurich (ETHZ). "In the current study, the researchers are converting cells derived from mesoderm into cells derived from ectoderm, and this is done without first wiping the cells' memory."

\section{Good behavior}

Wernig said his group is trying to replicate the findings in human cells. The team also wants to modify the protocol to generate other types of neuronal cells.

Jessberger also wants to see additional studies that characterize the behavior of neurons produced directly from fibroblasts. "What is unclear is how much of the fibroblast phenotype remains in these cells," he said.

"The work that needs to be done now is to show that this system functions with human cells and that human induced neuronal cells are representative of the actual cells taken from the body," Gregory told $S c i B X$.

"We don't have the full characterization of the epigenome of these cells," noted Berta Strulovici, CTO at iPS company iPierian Inc. She said this should be done if the cells are to be used for disease modeling.

"If the cells have the right physiology, they could become a powerful tool for studying developmental biology," said Strulovici.

Thomas Graf, program coordinator at the Center for Genomic Regulation, agreed that there needs to be more analysis of the functionality of the neurons produced by the Stanford researchers. He noted that his group has tried to directly convert fibroblasts into blood cells using transcription factors and ended up with cells that had many but not all functions that define macrophages. ${ }^{6}$

"What we got were cells that had macrophage characteristics but retained some fibroblast traits. However, we only used two transcription factors to switch the cells so there may be other transcription factors that are needed," he said.

\section{Brain frees}

In addition to characterizing the cells produced by the Stanford team's protocol, Graf said it will be important to show that transplanted 

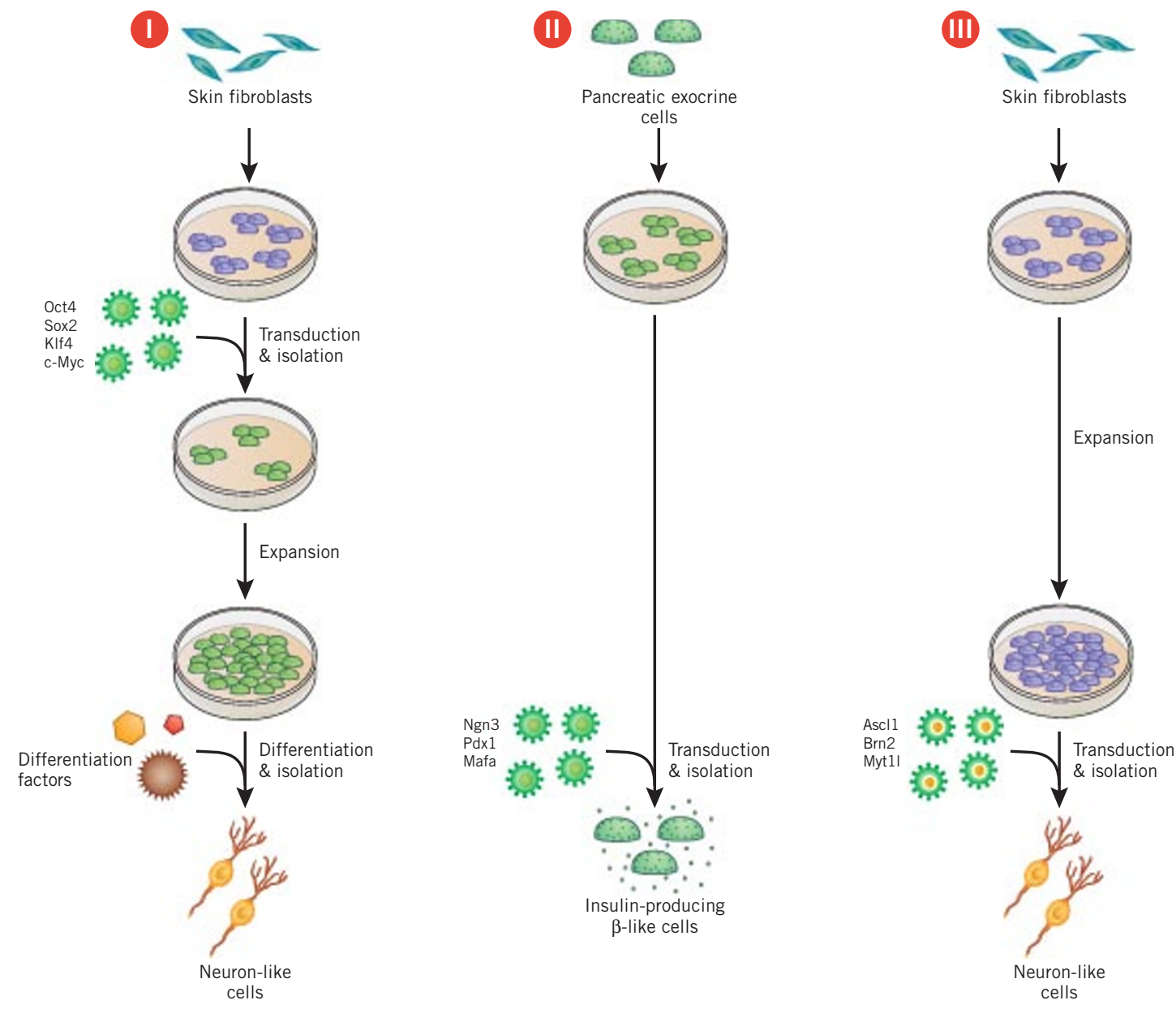

Figure 1. Generating neurons from fibroblasts. Researchers showed in Nature that fibroblasts can be directly converted into neurons without first reverting the skin cells to a pluripotent state. ${ }^{1}$

(I) Traditionally, researchers take isolated fibroblasts and use the transcription factors Oct4, Sox2, Klf4 and c-Myc to induce a pluripotent state. Cells that have been successfully reprogrammed are isolated and expanded in culture. Next, a cocktail of differentiation factors is added to convert the pluripotent cells into the desired cell type, which is then isolated from culture.

(II) Studies in 2008 showed that the forced expression of transcription factors can be used to interconvert cell types of the same lineage without first reverting them to a pluripotent state. ${ }^{4,5}$ In one such study, researchers transduced mouse pancreatic exocrine cells with neurogenin 3 (Ngn3), pancreatic and duodenal homeobox 1 (Pdx1) and v-maf musculoaponeurotic fibrosarcoma oncogene homolog A (Mafa) to convert them into insulin-producing $\beta$-like cells. ${ }^{5}$

(III) In the current study, researchers converted mouse fibroblasts into cells of an unrelated tissue lineage-neuronal cells. ${ }^{1}$ Isolated fibroblasts were expanded in culture and transduced with achaete-scute complex homolog 1 (Ascl1), POU class 3 homeobox 2 (Pou3f2; Brn2) and myelin transcription factor 1-like (Myt1l) using retroviral vectors. Cells successfully reprogrammed into neurons were then isolated from the culture.

induced neuronal cells can integrate and contribute to existing neuronal networks in animal models.

If this proves to be the case, said Jessberger, researchers would have a powerful new tool for disease modeling and drug screening.

"When it comes to diseases of the brain, the ability to study the cells taken from patients themselves cannot be underestimated. The mouse has served us greatly, but in complex neurological conditions like bipolar disorder, depression, schizophrenia, there really isn't a good animal model available," said Wernig.

Strulovici did note that scalability could become an issue for cells pro- duced by the Stanford protocol because fibroblasts are not immortal.

"It could be difficult to obtain a lot of cells from fibroblasts, and drug screening studies require a lot of cells," she told SciBX. "iPS cells have the advantage of scalability, which is very important for drug screening. These cells are immortal so you potentially have an unlimited supply of cells."

Stanford has filed a patent application covering the work and its use in undisclosed indications. The work is available for licensing.

Lou, K.-J. SciBX 3(7); doi:10.1038/scibx.2010.206

Published online Feb. 18, 2010 


\section{REFERENCES}

1. Vierbuchen, T. et al. Nature; published online Jan. 27, 2010; doi:10.1038/nature08797

Contact: Marius Wernig, Stanford University School of Medicine, Stanford, Calif.

e-mail: wernig@stanford.edu

2. Okita, K. et al. Nature 448, 313-317 (2007)

3. Takahashi, K. et al. Cell 131, 861-872 (2007)

4. Seale, P. et al. Nature 454, 961-967 (2008)
5. Zhou, Q. et al. Nature 455, 627-632 (2008)

6. Feng, R. et al. Proc. Natl. Acad. Sci. USA 105, 6057-6062 (2008)

COMPANIES AND INSTITUTIONS MENTIONED

Center for Genomic Regulation, Barcelona, Spain

iPierian Inc., South San Francisco, Calif.

Stanford University School of Medicine, Palo Alto, Calif.

Sangamo BioSciences Inc. (NASDAQ:SGMO), Richmond, Calif.

The Swiss Federal Institute of Technology Zurich, Zurich, Switzerland 